\title{
Rice DENSE AND ERECT PANICLE 2 is essential for determining panicle outgrowth and elongation
}

Feng $\mathrm{Li}^{1,2,{ }^{*}}$, Wenbo Liu ${ }^{1,2, *}$, Jiuyou Tang ${ }^{1,2}$, Jinfeng $\mathrm{Chen}^{1,2}$, Hongning Tong ${ }^{1,2}$, Bin $\mathrm{Hu}^{1,2}$, Chunlai $\mathrm{Li}^{1,2}$, Jun Fang ${ }^{1}$, Mingsheng Chen ${ }^{1}$, Chengcai Chu ${ }^{1}$

${ }^{I}$ State Key Laboratory of Plant Genomics and National Center for Plant Gene Research, Institute of Genetics and Developmental Biology, Chinese Academy of Sciences, Beijing 100101, China; ${ }^{2}$ Graduate School of the Chinese Academy of Sciences, Beijing 100101, China

The architecture of the panicle, including grain size and panicle morphology, directly determines grain yield. Panicle erectness, which is selected for achieving ideal plant architecture in the northern part of China, has drawn increasing attention of rice breeders. Here, dense and erect panicle 2 (dep2) mutant, which shows a dense and erect panicle phenotype, was identified. DEP2 encodes a plant-specific protein without any known functional domain. Expression profiling of $D E P 2$ revealed that it is highly expressed in young tissues, with most abundance in young panicles. Morphological and expression analysis indicated that mutation in DEP2 mainly affects the rapid elongation of rachis and primary and secondary branches, but does not impair the initiation or formation of panicle primordia. Further analysis suggests that decrease of panicle length in dep2 is caused by a defect in cell proliferation during the exponential elongation of panicle. Despite a more compact plant type in the dep2 mutant, no significant alteration in grain production was found between wild type and dep2 mutant. Therefore, the study of DEP2 not only strengthens our understanding of the molecular genetic basis of panicle architecture but also has important implications for rice breeding.

Keywords: panicle architecture; dense and erect panicle; rice

Cell Research (2010) 20:838-849. doi:10.1038/cr.2010.69; published online 25 May 2010

\section{Introduction}

Rice is one of the most important food crops in the world, feeding over half of the global population. The architecture of rice plants represents a combination of important and complex agronomic traits. Modification of plant architecture to create new elite cultivars is considered as a viable approach to increasing grain yield [1]. So far, numerous QTLs or genes controlling plant architecture have been cloned [2-5]. In the case of the 'Green Revolution', grain yields have been significantly increased by growing lodging-resistant semi-dwarf varieties of wheat and rice $[6,7]$.

\footnotetext{
*These two authors contributed equally to this work.

Correspondence: Chengcai Chu

Tel/Fax: +8610 64877570

E-mail: ccchu@genetics.ac.cn

Received 23 November 2009; revised 7 February 2010; accepted 8 March 2010; published online 25 May 2010
}

The architecture of the rice panicle is mainly determined by the arrangement of branches and spikelets. Panicle erectness, which is highly related to grain yield contributors, such as canopy shade areas and corresponding utilization efficiency of solar energy, and physiological conditions, such as humidity, temperature, and $\mathrm{CO}_{2}$ aeration, has increasingly drawn the attention of rice breeders $[8,9]$. Since the 1960 s, a number of highyielding japonica rice varieties have been released and predominantly cultivated in japonica rice planting areas ranging from the Yangtze River to Songliao Plain of China [9]. Most erect panicle varieties are derived from the cross of Balilla or Balilla-derived varieties with other parents [10]. Several groups have reported the mapping of this QTL to a similar location on chromosome 9 using different rice germplasms [11-13], and a gene named DENSE AND ERECT PANICLE 1 (DEP1)/qPE9-1 was recently cloned, encoding a PEBP (phosphatidylethalamine-binding protein)-like-domain protein $[10,14]$. Most recently, two other erect panicle genes, EP2 and EP3, 
have been reported; $E P 2$ was mapped to chromosome 4, and $E P 3$ encodes a putative F-box protein $[15,16]$.

The development of the rice panicle is a complicated process, which has been categorized into nine stages according to landmark events during the developmental course. Interestingly, rapid elongation of the panicle does not start until stage In 8 [17], when inflorescence meristems abort and all floral organs, like primary branches, secondary branches, and spikelets, are differentiated. Currently, several genes involved in the formation or initiation of floral meristems have been isolated [18-24]. MOC1 (MONOCULM 1) is required for both vegetative and reproductive axillary meristem formation [23]. The b-HLH transcriptional regulator, encoded by $L A X$ ( $L A X$ $P A N I C L E)$, is involved in the initiation/maintenance of the floral branch meristem [21]. FZP (FRIZZY PANI$C L E)$ is a positive regulator of floral meristem identity, suppressing the formation of axillary meristems of rice spikelets [22]. Rice FON1 is shown to be an ortholog of Arabidopsis CLV1, which controls floral meristem size and floral organ number [24]. APO1 (ABERANT PANICLE ORGANIZATION 1) participates in the formation of floral organ and primary branch phyllotaxy $[19,20]$. OsCKX2 (CYTOKININ OXYDASE/DEHYDROGENASE), which encodes a cytokinin oxidase, affects grain number by modulating the content of cytokinin, suggesting an important role of cytokinin in rice panicle development
[18]. Recently, SP1 (SHORT PANICLE 1) has been identified to encode a putative transporter that belongs to the peptide transporter (PTR) family and may function as a nitrate transporter. The spl mutant is defective in rice panicle elongation without affecting the initiation or formation of floral meristems [25].

Here, we report the study of two allelic dense and erect panicle mutants, designated as dep2-1 and dep2-2. Cloning and characterization of $D E P 2$ reveal that DEP2 is a novel protein, affecting the elongation of panicle branches.

\section{Result}

\section{Characterization of dep2}

To elucidate the molecular mechanism of panicle erectness, two dense and erect panicle mutant alleles, dep 2-1 and dep2-2, derived respectively from Oryza sativa L. ssp. japonica cultivar Zhonghua 11 and Nipponbare were identified [26]. Phenotypic analysis indicated that the morphology of dep2 was comparable with the wild type plant from the vegetative developmental stage to the early reproductive stage. However, wild type panicles begin to bend 3 weeks after flowering as grain weight increases, while the panicles of dep2 remained upright, even after the grains were fully matured (Figure $1 \mathrm{~A})$.
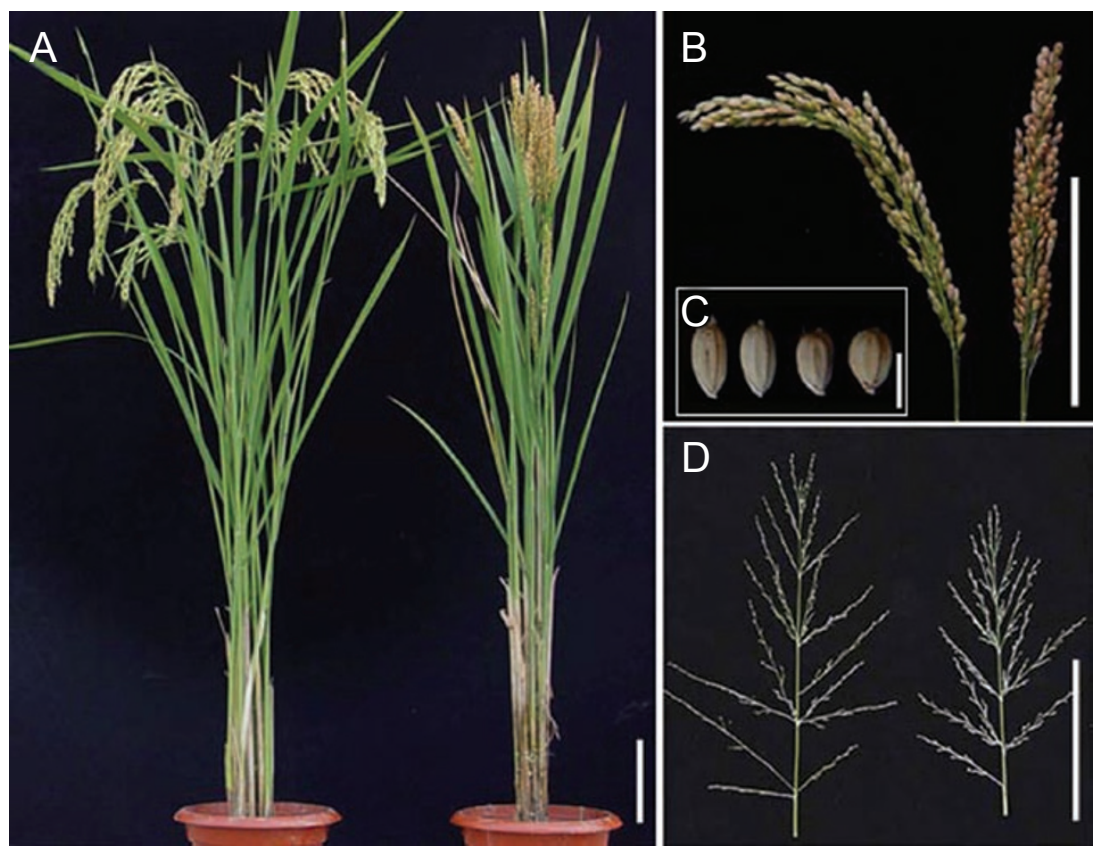

Figure 1 Phenotype of the dep2 mutant. (A, B) Gross morphology (A) and panicle morphology (B) of wild type (left) and dep2-1 (right) at mature stage. Bar $=10 \mathrm{~cm}$. (C) Comparison of the mature grains between the wild type (left) and dep2-1 (right). Bar $=5 \mathrm{~mm}$. (D) Comparison of the panicle branching between the wild type and dep2-1. Bar $=10 \mathrm{~cm}$. 
Besides panicle erectness, the dep 2 mutants also showed a slight reduction in plant height (Figure 1A and Table 1), an obvious decrease in panicle length (Figure 1B), and a significant increase in both rachis and stem diameter (Table 1). The leaves of dep2 are short, wide, and erect, and the overall appearance of the mutant is more compact compared to the wild type (Supplementary information, Figure S1). Detailed analysis showed that there were no difference in the number of primary and secondary branches (Figure 1D), and the total number of spikelets per panicle between the wild type and the mutant (Table 1). The grain density is increased due to the decreased panicle length but not the change of grain number. The grains of the mutant are wider and shorter than the wild type (Figure 1C), causing a slight decrease in the 100-grain weight, $2.51 \mathrm{~g}$ in dep2-1 in contrast to $2.72 \mathrm{~g}$ in Zhonghua11 (Table 1). These results indicate that mutation in DEP2 has pleiotropic effects on plant architecture, and that increased diameter of the rachis and decreased panicle length altogether contributed to the dense and erect panicle phenotype.

The dep2 mutant is defective in the elongation of young panicles

Scanning electron microscope (SEM) observation of the developmental course of the panicle was carried out to examine the defects in dep 2 panicles. No significant difference could be observed between the wild type and the mutant at early developmental stages, including the generation of the first and second bract (Supplementary information, Figure S2A, S2E), and formation of primary and secondary branch primordia (Supplementary information, Figure S2B, S2F and S2C, S2G) and flower organs (Supplementary information, Figure S2D, S2H). We could not find any difference when the panicle of wild type grew to $1 \mathrm{~cm}$ long (Figure 2A); however, the panicle length of the mutant was reduced by about $30 \%$ when the panicle of the wild type was $5 \mathrm{~cm}$ long (Figure 2B). The delayed growth lasted through the rest phase of panicle development, leading to the reduced panicle length (Figure 2C-2E). These results are consistent with the developmental course of the florets (Figure 2F-2J). Development of rice inflorescence is categorized into nine stages. Proximal primary branch primordia, in spite of earlier formation, seem not to elongate until the last primordium is formed, and all the primordia almost simultaneously start to elongate at In 8 , when the length of inflorescences reaches $40 \mathrm{~mm}$ and differentiation of all floral organs is finished [17]. On the basis of these categories, we may conclude that the erect panicle in dep2 was caused during the late stage of panicle development, while the formation of primordia and differentiation of spikelets were not affected in young panicle development.

To investigate whether the defect in the elongation of the inflorescences in dep 2 mutant was caused by abnormal cell elongation and/or cell proliferation, we compared the longitudinal sections of uppermost internodes, rachis axis, and florets at the late stage of heading. No difference could be detected in cell length in any of the three organs between the wild type and the mutant (Figure $3 \mathrm{~A}-3 \mathrm{~F}$ ), suggesting that decrease of the panicle length may be caused by a defect in cell proliferation. The expression levels of cell cycle-related genes $C y c B 1 ; 1$, $C y c B 2 ; 1, C y c B 2 ; 2, C y c D 3 ; 1$, and $C D K B 2 ; 1$ were all decreased in the mutant compared to the wild type (Supplementary information, Figure S3).

\section{Cloning of the DEP2 gene}

Genetic analysis revealed that dep2-1 and dep2-2 are allelic to each other and are formed by single recessive nuclear gene mutations (data not shown). Map-

Table 1 Morphometric analysis of wild type and dep 2 plants

\begin{tabular}{|c|c|c|c|c|}
\hline Traits & Wild type (Zhonghua 11) & dep2-1 & Wild type (Nipponbare) & dep2-2 \\
\hline Plant height $(\mathrm{cm})$ & $104.6 \pm 4.4$ & $88.4 \pm 3.8^{* * *}$ & $93.9 \pm 2.6$ & $70.9 \pm 4.4^{* * *}$ \\
\hline Panicle length (cm) & $23.3 \pm 1.2$ & $16.87 \pm 0.9 * * *$ & $19.6 \pm 1.0$ & $13.5 \pm 0.9 * * *$ \\
\hline NPB (No.) & $13.3 \pm 1.6$ & $13.9 \pm 1.5$ & $9.8 \pm 0.5$ & $9.8 \pm 0.8$ \\
\hline NSB (No.) & $38.4 \pm 8.9$ & $38.5 \pm 7.1$ & $17.6 \pm 2.6$ & $16.7 \pm 2.5$ \\
\hline 100-grain weight (g) & $2.72 \pm 0.01$ & $2.51 \pm 0.03 * * *$ & $2.26 \pm 0.05$ & $2.12 \pm 0.01 * *$ \\
\hline
\end{tabular}

NPB, number of primary branches per panicle; NSB, number of secondary branches per panicle; SN, number of spikelets per panicle. Data are averages of 15 plants $( \pm \mathrm{SD})$. Asterisks indicate the significance of differences between wild type and dep2 plants, as determined by Student's $t$-test: $* * 0.001 \leq P<0.01, * * * P<0.001$. 

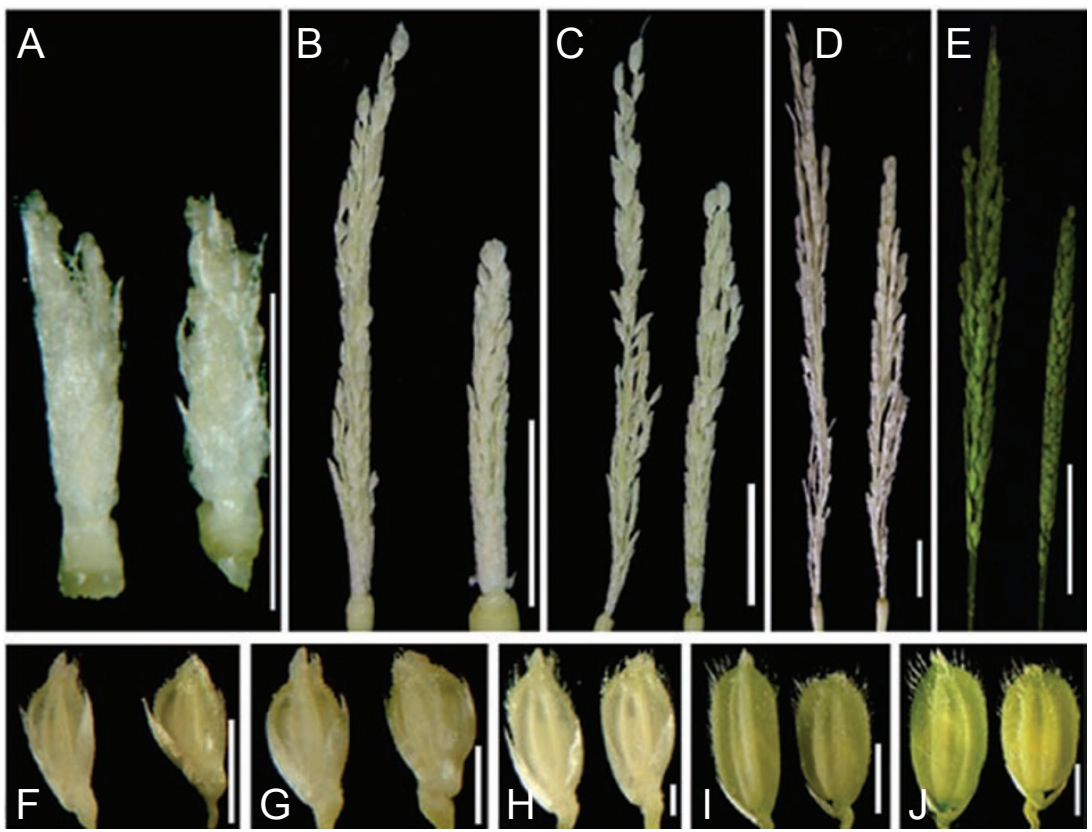

Figure 2 Comparison of the panicle development between the wild type and dep2-1. (A-E) Panicles of the wild type (left) of 1 $\mathrm{cm}(\mathbf{A}), 5 \mathrm{~cm}$ (B), $10 \mathrm{~cm}$ (C), $15 \mathrm{~cm}$ (D), and $20 \mathrm{~cm}$ (E) and of dep2-1 (right) at the same stage. (A-D) Bar = $1 \mathrm{~cm}$; (E) Bar = 5 $\mathrm{cm}$. (F-J) Florets of the wild type (left) panicle of $3 \mathrm{~cm}(\mathbf{F}), 5 \mathrm{~cm}(\mathbf{G}), 10 \mathrm{~cm}(\mathbf{H}), 15 \mathrm{~cm}$ (I), and $20 \mathrm{~cm}(\mathrm{~J})$ and dep2-1 (right) at the same stage. $(\mathbf{F}-\mathrm{H}) \mathrm{Bar}=100 \mu \mathrm{m} ;(\mathbf{I}, \mathbf{J}) \mathrm{Bar}=2 \mathrm{~cm}$.
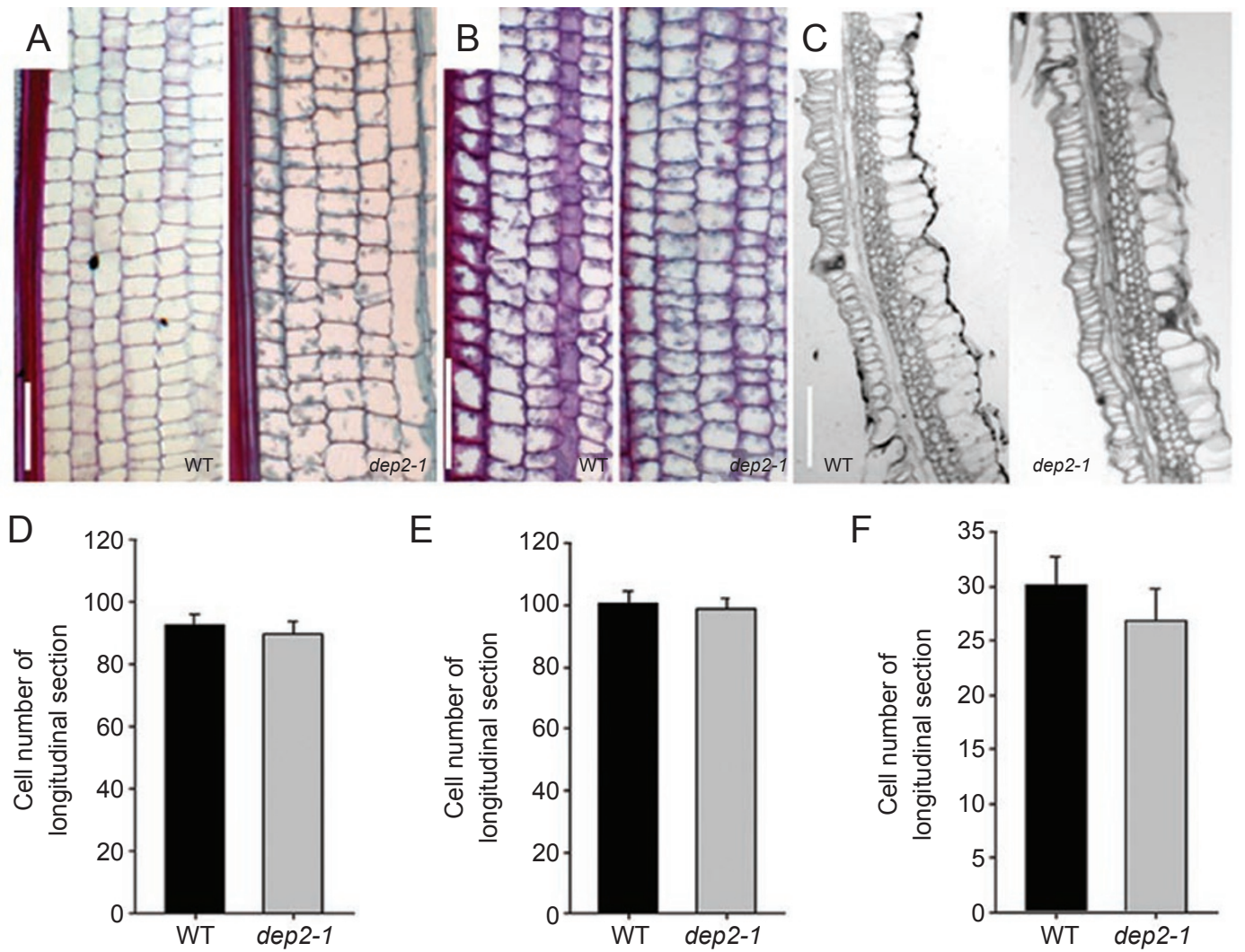

Figure 3 Histological analysis. (A, D) Comparison of the longitudinal section of the uppermost internodes between the wild type and dep2-1. Bar $=100 \mu \mathrm{m}$. (B, E) Comparison of the longitudinal section of the rachis axis between the wild type and dep2-1. Bar $=100 \mu \mathrm{m}$. (C, F) Comparison of the longitudinal section of the florets between the wild type and dep2-1. Bar $=100 \mu \mathrm{m}$. 
ping populations were constructed respectively based on crosses between the mutants and an indica cultivar Minghui 63 . The DEP2 locus was mapped to the long arm of rice chromosome 7 between markers M1 and M5 (Figure 4A). We developed other three molecular markers and further delimited the target gene to a 27 $\mathrm{kb}$ region by markers M2 and M15 (Figure 4A). Within this 27-kb interval, there are five predicted ORFs: LOC Os07g42390, LOC_Os07g42395, LOC_Os07g42400, LOC_Os07g42410, and LOC_Os07g42420 (Figure 4B). DNA sequence comparison revealed a 31-bp deletion in the sixth exon and a G/A substitution in the second intron of LOC_Os07g42410 from dep2-1 and dep2-2, respectively (Figure 4C), and no sequence difference was found in other predicted ORFs. The 31-bp deletion in dep2-1 starts at $2184 \mathrm{bp}$ from the initiation codon ATG and caused a frameshift; the G/A substitution in the second intron of dep2-2 caused an altered splicing site of the second intron and also led to a frameshift. Thus LOC Os07g 42410 was considered a candidate for the $D E P 2$ gene.

An $11.1 \mathrm{~kb}$ genomic fragment containing the entire $D E P 2$ coding region, 2397 bp $5^{\prime}$ upstream sequence, and $1335 \mathrm{bp} \mathrm{3'}$ downstream region, was constructed into the binary vector pCAMBIA1300 and transformed into the dep2-2 background. As a control, the pCAMBIA1300
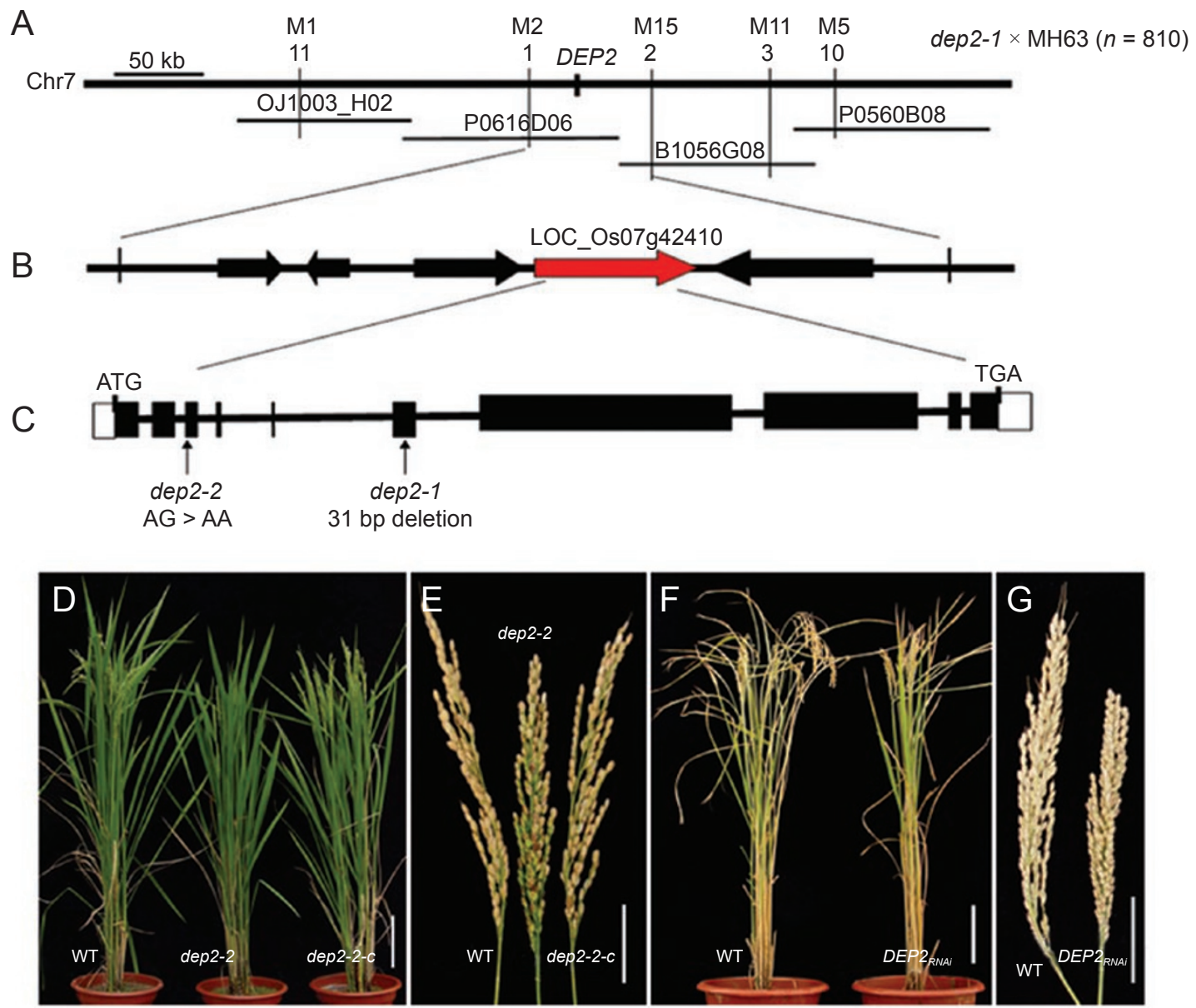

Figure 4 Map-based cloning of the DEP2 gene. (A) The DEP2 locus was mapped to the long arm of rice chromosome 7 between markers M1 and M5. The gene was further delimited to a $27 \mathrm{~kb}$ genomic region between the markers M2 and M15 within the BAC clone P016D06 and B1056G08. The number of recombinants is marked corresponding to the molecular markers. (B) Within this 27-kb interval, there are five predicted ORFs and the candidate gene is marked in red. (C) Schematic representation of the DEP2 gene structure. Black boxes indicate the coding sequence, white boxes indicate the 5' and 3' untranslated regions, and lines between boxes indicate introns. Mutation being identified in dep2-1 and dep2-2 are indicated by arrows. (D, E) Complementation analysis of the dep2-2 mutant. (D) Gross morphology at the heading stage and (E) the panicle morphology at mature stage. (D) Bar $=10 \mathrm{~cm}$; (E) Bar $=4 \mathrm{~cm}$. (F, G) RNAi analysis of the DEP2 gene. Gross morphology (F) and panicle morphology (G) at mature stage. $(\mathbf{F})$ Bar $=10 \mathrm{~cm} ;(\mathbf{G})$ Bar $=4 \mathrm{~cm}$. 
vector was also introduced into dep2-2. We found that the dep2-2 mutant phenotype was rescued in transgenic plants carrying the candidate gene (Figure 4D and 4E). $D E P 2$ knockdown transgenic lines were generated by introducing the specific RNA interference (RNAi) construct into wild type plants and the transgenic plants mimicked the dep 2 mutant phenotype (Figure $2 \mathrm{~F}$ and $2 \mathrm{G})$. Therefore, we conclude that mutation of $D E P 2$ gene is responsible for the altered phenotype of $\operatorname{dep} 2$.

DEP2 encodes a novel plant-specific protein

The $D E P 2$ gene is predicted to encode a 1365 amino acid protein with a pI of 6.23 and molecular mass of 149
$\mathrm{kDa}$. To gain further insight into the possible function of the DEP2 protein, its sequence was used to search Protein Families database of alignments and hidden Markov models [27] and other public databases. Surprisingly, DEP2 does not possess any known protein domains, indicating this protein is novel. Searching of the GenBank database (http://www.ncbi.nlm.nih.gov/BLAST) and JGI (http://genome.jgi-psf.org) identified several putative DEP2 homologs from rice (O. sativa L.), Arabidopsis, poplar (Populus trichocarpa), grape (Vitis vinifera), maize (Zea mays), and sorghum (Sorghum bicolor), and 26 sequences from these plant species were adopted to construct a phylogenetic tree (Figure 5).

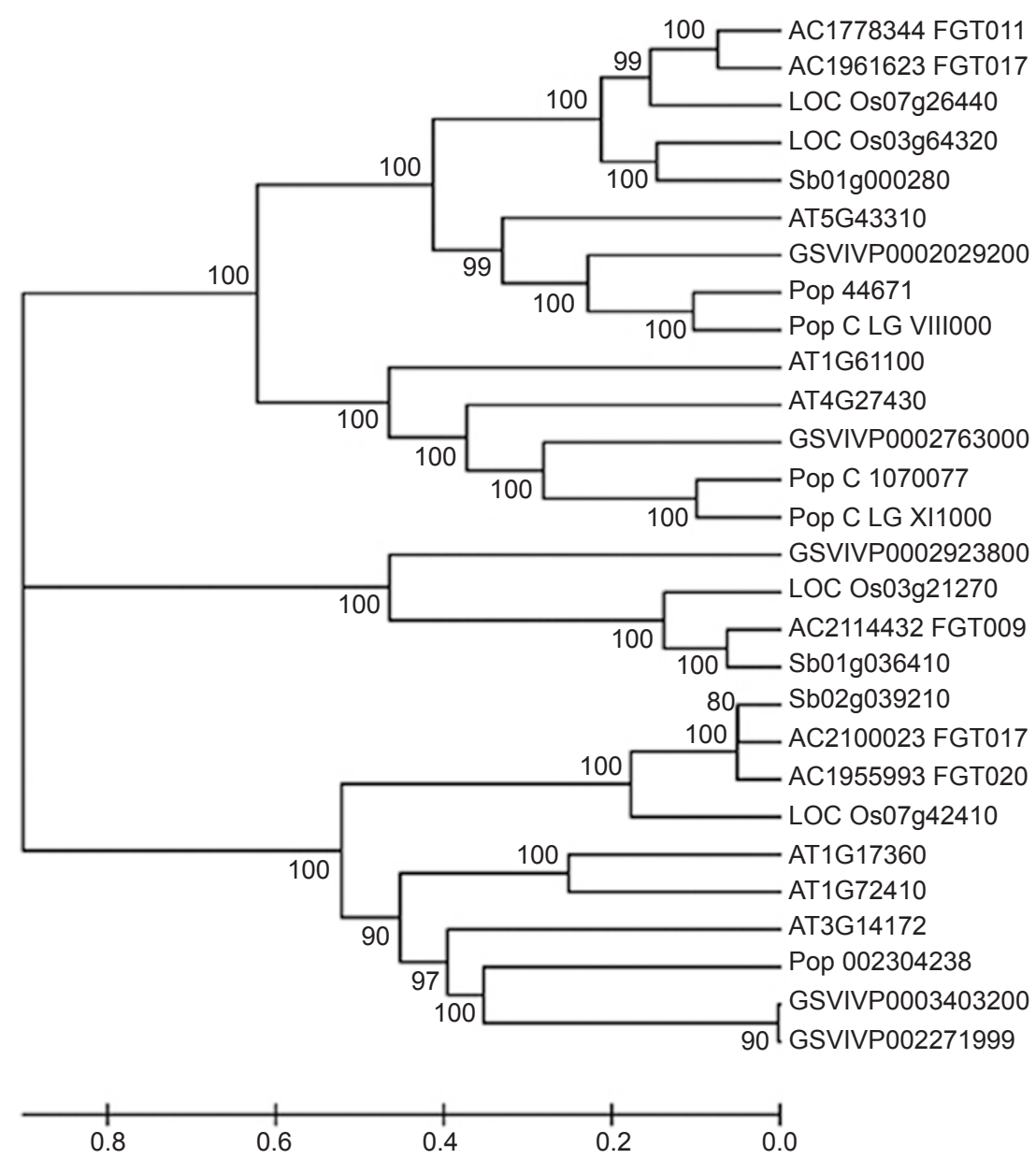

Figure 5 Phylogenetic analyses of putative homologs of DEP2. Phylogenetic analysis of putative DEP2 homologs using MEGA with neighbor-joining method; bootstrap analysis was performed with 1000 replicates and excluding positions with gaps. Numbers in branches indicate bootstrap values (percent). Homologous genes are with the following accession numbers: Oryza sativa (LOC_Os03g21270, LOC_Os07g26440, LOC_Os03g64320, and LOC_Os07g42410); Arabidopsis (AT1G611001, AT4G274301, AT1G173601, AT1G724101, AT3G141721, and AT5G433101); Populus trichocarpa (Pop_44671, Pop_C_LG_VIII000, Pop_C_1070077, Pop_C_LG_XI1000, and Pop_002304238); Vitis vinifera (GSVIVP0002029200, GSVIVP0002763000, GSVIVP0002923800, and GSVIVP0003403200); Zea mays (AC1955993_FGT020, AC2100023_FGT017, AC2114432_FGT009, AC1778344_FGT011, and AC1961623_FGT017); and Sorghum bicolor (Sb01g000280, Sb01g036410, and $\mathrm{Sb02g039210).}$ 

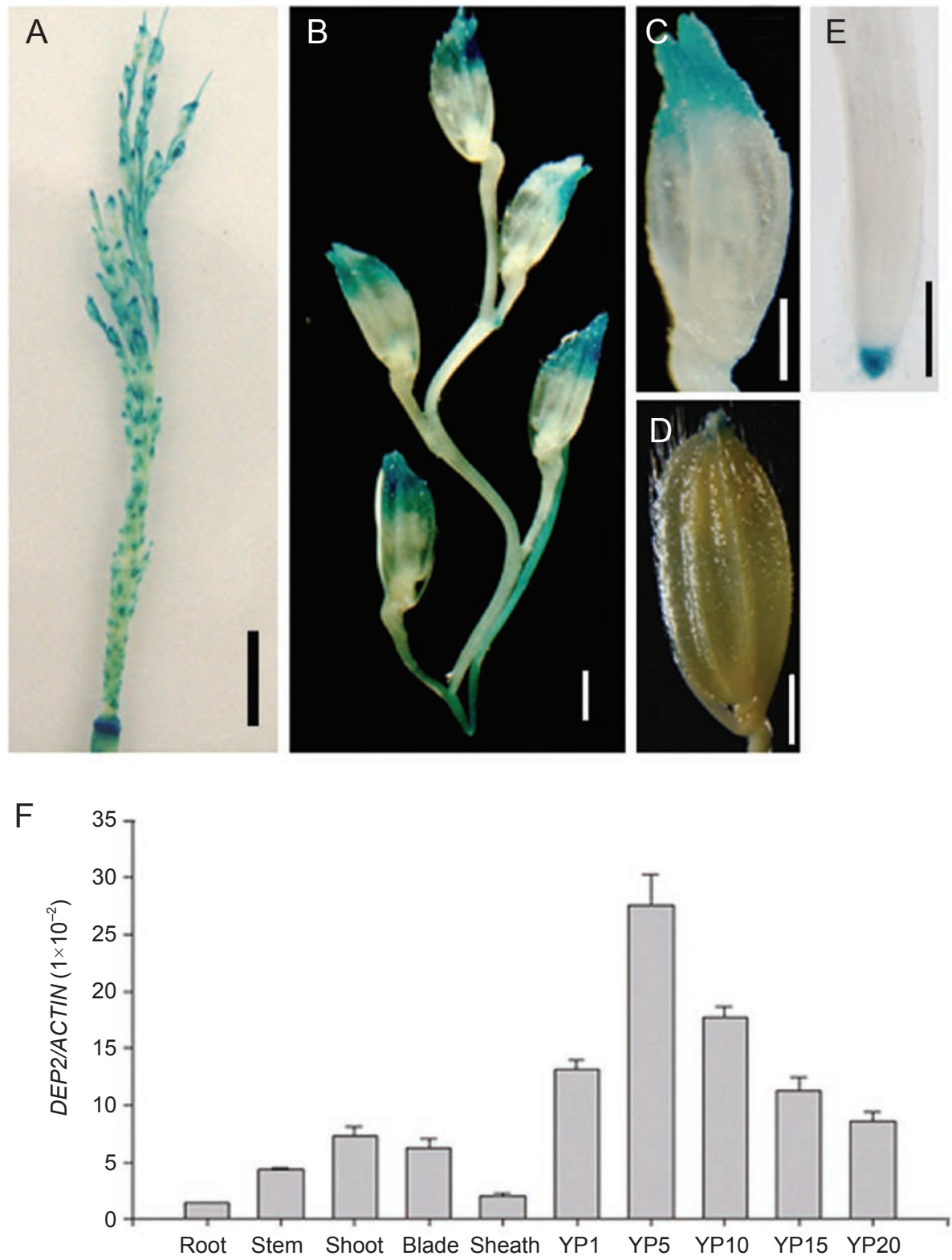

Figure 6 DEP2 expression pattern. (A-G) DEP2 expression pattern was revealed by the transformants with $D E P 2$ promoter GUS. GUS staining is found in young panicles (A), young florets (B, C), and in root (E), but not in old panicles (D). (A) Bar = $1 \mathrm{~cm}$; (B, C , E) Bar = $1 \mathrm{~mm}$; (D) Bar = $2 \mathrm{~cm}$. (H) Quantitative RT-PCR analysis of DEP2 expression in various organs, including root, stem, shoot, leaf blade, leaf sheath, and young panicle of $1 \mathrm{~cm}$ (YP1) to $20 \mathrm{~cm}$ (YP20).

Notably, three putative homologs, Sb02g039210, AC2100023 FGT017, and AC1955993 FGT020, which shared around $60 \%$ amino acid sequence identity with the DEP2 protein, were identified in the sorghum and maize genome, suggesting that the function of DEP2 homologs is conserved in monocot. There are also three putative homologs At3g14172, At1g17360, and At1g72410 in Arabidopsis and one from grape (GSVIVP0002763000), which shared around $40 \%$ amino acid sequence identity with the DEP2 protein.
There are also some other homologous proteins identified with low sequence identity with DEP2, including CIP7 (At4g27430), which is a COP1-interacting protein. CIP7 is a nuclear protein, which contains transcriptional activation activity and acts as a positive regulator of light-regulated genes [28]. However, the similarity between DEP2 and CIP7 is only restricted to the Nterminal part of the protein without any functional motif of CIP7. Yeast two-hybrid analysis was carried out to test the relationship between rice COP1 and DEP2, but no 
interaction could be detected in vivo; additionally, we did not find any transcriptional activation activity of DEP2 in contrast to CIP7 (data not shown). Proteins with weak similarities to DEP2 are also present in gymnosperm plant Picea sitchensis (ABR16652), and green algae, Physcomitrella patens subsp. patens (XP_001780764), but not in yeast or animals (data not shown). These data suggest that DEP2 is plant specific, and the existence of DEP2 proteins in different plant species might suggest its conserved biological function.

Expression pattern and subcellular localization of DEP2

To have a better understanding of $D E P 2$ function, we examined its spatial and temporal expression pattern by using the GUS reporter system. Approximately $2 \mathrm{~kb}$ of the $D E P 2$ upstream sequence was amplified and introduced into the pCAMBIA1391Z vector, resulting in the $P R O_{D E P 2}: G U S$ construct. Analysis of transgenic plants harboring the $P R O_{D E P 2}: G U S$ construct indicated the universal expression of $D E P 2$ in various tissues, but with preferential expression in actively dividing zones (Figure $6 \mathrm{~A}-6 \mathrm{C}, 6 \mathrm{E})$. The GUS signal was stronger in rachis, branches, and florets of the dividing zones than the other parts. We also noticed that the signal became weaker as panicles grew longer (Figure 6A-6C), and was barely detectable when panicles reached their final lengths (Figure $6 \mathrm{D})$. In the root, the $D E P 2$ promoter was active in root tips (Figure 6E).

Further quantitative RT-PCR analysis indicated that $D E P 2$ was highly expressed in young panicles ranging from 1 to $15 \mathrm{~cm}$ in length; low level expression was also detected in the other organs, including roots, stems, leaves, and leaf sheathes. The expression level reached to a peak when the panicle was about $5 \mathrm{~cm}$ long and decreased to a low level when the panicle length reached $\sim 20 \mathrm{~cm}$ (Figure 6F). This expression pattern correlates well with the panicle development and expression pattern obtained from the GUS reporter system, suggesting that DEP2 is required in the early rapid elongation stage of rice panicle.

We also examined the subcellular distribution of the DEP2 protein. Full-length $D E P 2$ coding sequence was fused in-frame to the $5^{\prime}$ end of the GFP gene under the control of the cauliflower mosaic virus $35 \mathrm{~S}$ promoter, and the construct was introduced into onion epidermal cells and Nicotiana benthamiana leaves via bombardment and infiltration, respectively. The signal of DEP2-GFP fusion protein could be detected in the cytoplasm, plasma membrane and nucleus in both onion epidermal cells (Figure $7 \mathrm{~A}$ ) and $N$. benthamiana leaves (Figure 7B). Thus, DEP2 appears to be ubiquitously distributed in plant cells.

\section{Discussion}

Previous studies have shown that extinction coefficient ( $k$-value) of canopy increases as rice panicle starts to bend at the middle stage of grain filling, influencing the top three leaves that contribute the most to the productivity. One important limit of IR8, which was first released in 1966, is the excessive mutual shading, which causes photosynthesis reduction in the canopy [29]. Erect panicle, reducing shade area in the canopy, thus has been
A

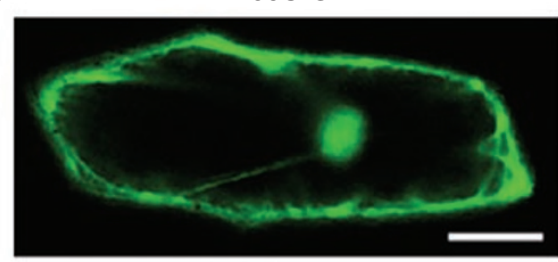

B

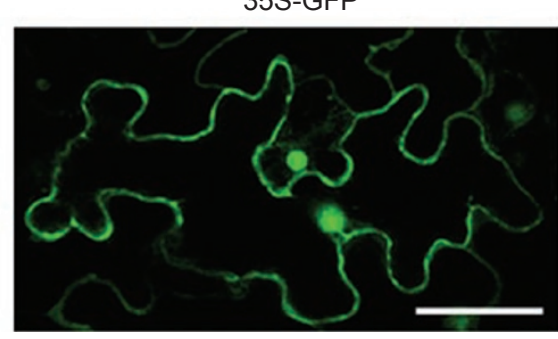

35S-GFP

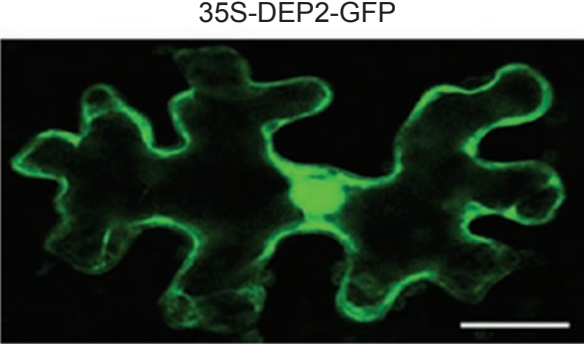

Figure 7 Subcellular localization of DEP2. (A) Localization in onion epidermal cells of 35S-GFP (left) and 35S-DEP2-GFP (right). Bar $=50 \mu \mathrm{m}$. (B) Localization of 35S-GFP (left) and 35S-DEP2-GFP (right) in epidermal cells of Nicotiana benthamiana leaves. Bar $=25 \mu \mathrm{m}$. 
taken as one of the most important agronomic traits in developing high-yield rice varieties. The release of Italian cultivar Balilla and derived commercial varieties with erect panicles like Shennong 265 and Jiahua 1, have dominated high-yield japonica rice acreage. Further, erect panicle is considered to be the third landmark trait (after dwarf and hybrid rice) in the history of Chinese rice breeding $[8,9,12,14]$.

In this study, we report the identification and characterization of the rice dep 2 mutant that displays dense and erect panicle phenotype. Morphological analysis showed that the dense and erect panicle phenotype in dep 2 mutants resulted from the decrease in the length of the rachis, primary and secondary branches, and the increase in the diameter of the rachis axis. In addition, plant height, culm diameter, and leaf morphology were also altered, resulting in a compact stature in the dep 2 mutants. And the increased diameter of both culm and panicle may help to enhance the potential in lodging and fertilizer resistance. Genetic studies of erect panicle varieties derived from Balilla and other mutants have identified panicle erectness genes, such as DEP1/qPE9-1, EP2, and $E P 3$, in recent years [10-12, 14-16]. Unlike dep 1 , the number of spikelets and primary and secondary branches was almost the same in dep 2 compared to the wild type.

Rice undergoes a series of complicated events to form the panicle structure. The first step is the formation of rachis meristem, arising from the shoot apical meristem. Primary and secondary branch meristems are then successively formed. The third step is the differentiation of floral organs such as glume, lodicules, stamen, and carpel. After all floral organs are differentiated, rachis and branches begin to elongate exponentially till heading, reaching the full length [17]. To date, most of the identified genes affecting panicle architecture are involved in the establishment of inflorescence meristems, while little is known about the molecular basis of the outgrowth and elongation of branches. SP1 (SHORT PANICLE 1), which encodes a putative PTR family transporter, was recently cloned. Growth and elongation of panicle are significantly delayed in the $s p 1$ mutant, leading eventually to arrested and faded branches after heading [25].

Our results demonstrate that the formation of primordia was not affected in dep2. No noticeable difference could be observed at the early stage of panicle elongation, but when the panicle grew $5 \mathrm{~cm}$ long, the panicle of the mutant was distinctively shorter than the wild type. We further compared the longitudinal section of the uppermost internodes, rachis axis, and florets at the last stage of heading and found that cell elongation was not affected, indicating that the phenotype (shortened panicle) might be caused by a defect in cell prolifera- tion. The expression levels of cell cycle-related genes $C y c B 1 ; 1, C y c B 2 ; 1, C y c B 2 ; 2, C y c D 3 ; 1$, and $C D K B 2 ; 1$ were all decreased in the mutant compared to the wild type (Supplementary information, Figure S3). Consistent with its function, $D E P 2$ expression is most abundant when the panicle is $5 \mathrm{~cm}$ long, at which time the panicle undergoes rapid elongation. In addition, GUS activity was mainly detected in the actively dividing region, especially in young panicles, and was barely detectable when the panicle reached its final size. These results suggest that DEP2 may be essential in panicle outgrowth and elongation instead of the initiation and formation of panicle primordia.

The map-based cloning of $D E P 2$ revealed that it encodes a large protein with no recognizable functional domain. DEP2 homologs were found from green algae to higher plants, but not in animals or fungi, suggesting that DEP2 may be a plant-specific protein. The only existing low similarity to CIP7 in Arabidopsis is found at the Nterminal part of the protein, while the COP1-interacting motif, transcription activation domain, and the nuclear localization signal domain are all missing in DEP2. By using the yeast two-hybrid system, we could not detect an interaction between PEP2 and COP1; and further, DEP2 did not show transcriptional activation activity in vivo (data not shown). Transient expression of DEP2GFP in both onion epidermal cells and tobacco leaves revealed that DEP2 shows a different subcellular localization pattern from that of COP1. These results suggest that the biological function of DEP2 may be different from CIP7.

Erect panicle trait has been used in rice breeding programs in the northern part of China, resulting in marked increases in grain yield. The extensive use of limited erect panicle sources, mainly derived from Balilla, however, causes a bottleneck effect in the genetic background when breeding for new varieties, and this may cause an eventual genetic vulnerability of crops to pests and diseases [30]. Although the reduced grain length leads to a slight decrease in 100-grain weight, dep 2 optimizes canopy structure and increases lodging and fertilizer resistance; hence, the new $D E P$ gene identified in this study may provide another candidate for future molecular breeding. Further studies to clarify the molecular mechanism of DEP2 should help shed light on the developmental processes that underpin rice panicle formation.

\section{Materials and Methods}

\section{Plant materials and growth conditions}

The rice (O. sativa L.) dep2-1 (japonica cv. Zhonghua 11) and dep2-2 (japonica cv. Nipponbare) mutants were isolated from our 
T-DNA insertion population. Rice plants were cultivated in the experimental field at the Institute of Genetics and Developmental Biology in Beijing, during the natural growing season.

\section{Map-based cloning}

The dep2-1 mutant was crossed with Minghui 63, an indica variety. Plants showing dense and erect panicle phenotype in the F2 progeny were selected for the genetic linkage analysis. Molecular makers distributed throughout the rice genome were utilized for preliminary mapping [31, 32]. Additional STS markers were designed according to the DNA sequences of indica and japonica (http://www.ncbi.nlm.nih.gov). Primer pairs used were as follows: MP1 (5'-TAC CTC TTC CGT TCA CTG-3' and 5'-TAC GTT TAC TTT GTT CAT CT-3'), MP2 (5'-AGG AGC CCA TCC GAT CTT CT-3' and 5'-GGA GCA GCG CTA GGG TGA G-3'), MP5 (5'CAT GAA CCT TTT GCA TTT-3' and 5'-TTG GCT ATA CTA TTG AAC CTG-3'), MP11 (5'-CAA CCG AAT CCA AAG TCA-3' and 5'-AAC GGA ACT CAA CTC ACC A-3'), and MP15 (5'-ACT GAT TCC GCA TTA TTT G-3' and 5'-TAG TGG CGG TAG AGG TAC-3').

\section{Complementation test}

For complementation of dep 2 mutant, $11.1 \mathrm{~kb}$ fragment containing the entire DEP 2 coding region, the 2 798-bp upstream sequence, and the 1335 -bp downstream sequence was acquired from BAC a0079J03 digested with the restriction enzyme BglII/ SwaI. The fragment was inserted into pCAMBIA1300 vector carrying a hygromycin-resistant gene, by digesting with $B g l \mathrm{II} / \mathrm{SmaI}$ to generate the transformation plasmid for the complementation test. The resulting transformation plasmid, as well as the empty pCAMBIA1300 vector as control, was introduced into the dep2-2 mutant by Agrobacterium tumefaciens-mediated transformation according to a published protocol [33].

\section{RNAi construct}

RNAi construct was constructed as described previously [34]; briefly, the OSGRF fragment in pCGI was replaced with the ORF fragments of $D E P 2$ (p4501 targeting to +2875 to $+3203 \mathrm{bp}$ ) by the primer 5'-CTC GAG AAA ATA CAA AGC CCT CAG-3' and 5'-AGA TCT AAT CCA GCT ATA CCG ACA-3'. DNA fragments that consist of a sense and an antisense strand separated by an intron were inserted into pXQ35S (a derivative of pCAMBIA2300 carrying the CaMV $35 \mathrm{~S}$ promoter and the OCS terminator).

\section{Scanning electron microscopy}

Shoot apexes of the wild type and dep 2 plants were collected on a daily basis from the vegetative stage, shortly before phase transition to the end of floral differentiation. Shoot apexes were dissected carefully and fixed overnight at $4{ }^{\circ} \mathrm{C}$ in FAA (formalin: glacial acetic acid: $70 \%$ ethanol; 1:1:18), and dehydrated in a graded ethanol series. The samples were dried in a critical-point drier, sputter-coated with platinum, and observed under a SEM (Quanta200; FEI, http://www.fei.com/).

\section{Histological analysis}

For microscopic observation, uppermost internodes, rachis axis, and florets in the late stage of heading were fixed in FAA (formaldehyde: glacial acetic acid: $70 \%$ ethanol; $1: 1: 18$ ) and dehydrated in a gradient ethanol series. The samples were embedded in Paraplast Plus (Sigma). Microtome sections of $10 \mu \mathrm{m}$ thickness were applied to silane-coated glass slides (Sigma). Paraffin was removed from the sections using xylene, and then the sections were dehydrated through a gradient ethanol series, and stained with toluidine blue before observation.

\section{RNA extraction and quantitative RT-PCR}

Total RNA was extracted using the guanidinium isocyanate/ acidic phenol method described previously [35]. The RNA was pre-treated with DNase I, and first-strand cDNA was synthesized from $2 \mu \mathrm{g}$ total RNA using oligo $(\mathrm{dT})_{18}$ as primers. First-strand cDNA product equivalent to $50 \mathrm{ng}$ total RNA was used as template in a $20 \mu \mathrm{l} \mathrm{PCR}$ reaction. For quantitative RT-PCR, SYBR Green I was added to the reaction system and run on a Chromo 4 real-time PCR detection system (Bio-Rad, http://www.bio-rad. com/) according to the manufacturer's instructions. The data were analyzed using Opticon monitor software (Bio-Rad). Three repeats were carried out for each gene. The rice ACTIN1 gene was used as an internal control in the analysis (primer pairs 5'-ACA TCG CCC TGG ACT ATG ACC A-3' and 5'-GTC GTA CTC AGC CTT GGC AAT-3'). The primers for quantitative RT-PCR analysis of DEP2 expression were 5'-TGC GTG ATA GCC TAG AAC GAA G-3' and 5'-CTG GAA TCA GCA CTC CTG GAT G-3'; for $C y c B 1 ; 1$ they were $5^{\prime}$-AGG TTG CTG CCT CTG CTG TCT A-3' and 5'GTG AGC GAA GTG CCA CTC CTC C-3'; for CycB2;1, 5'-CTT GAG TCA GGA GCA GGA GGT-3' and 5'-AGC TTA CAT AGG CTG AAT GCC-3'; for $C y c B 2 ; 2,5^{\prime}$-GGC ACT GTA ACT GAT TGG CTC A-3' and 5'-CAA ACG CAG ATC AAT GTC TCG-3'; for $C y c D 3 ; 1,5^{\prime}-\mathrm{CCC}$ CAA GGA TGA GAT GGC AGA G-3' and 5'-ACG AGC TGT CGC AGC TGA AGC-3'; and for CDKB2;1, 5'-AAG CAG GGG CAG AAC AAG GAG G-3' and 5'-TGG TCT TGC GGT CCA TGA GCA G-3'.

\section{GUS staining}

For promoter analysis, about $2 \mathrm{~kb}$ of $D E P 25^{\prime}$ region was amplified with primers $5^{\prime}$-ACA AGC TCC CTT GGT TGC A-3' and 5'-CGA GGT CGG ATC TGG TGG A-3', and inserted into the SalI and EcoRI site of pCAMBIA1391Z vector. The resulting plasmid was transformed into rice, and GUS staining was performed according to the method described previously [36]. Various tissues or hand-cut sections of $P R O_{\mathrm{DEP} 2}: G U S$ transgenic plants were incubated in a solution containing $50 \mathrm{mM} \mathrm{NaPO}_{4}$ buffer ( $\left.\mathrm{pH} 7.0\right), 5$ $\mathrm{mM} \mathrm{K}_{3} \mathrm{Fe}(\mathrm{CN})_{6}, 5 \mathrm{mM} \mathrm{K}_{4} \mathrm{Fe}(\mathrm{CN})_{6}, 0.1 \%$ Triton X-100, and $1 \mathrm{mM}$ $\mathrm{X}$-Gluc at $37{ }^{\circ} \mathrm{C}$. Images were taken directly or under the stereomicroscope (SZX16, Olympus, http://www.olympus-global.com/).

\section{Phylogenetic analysis}

Homolog sequences of DEP2 in Populus trichocarpa, Sorghum bicolor, and Vitis vinifera were obtained at JGI website (http:// genome.jgi-psf.org), other homologous sequences were obtained from PsiBlast searches at the National center for Biotechnology Information (http://blast.ncbi.nlm.nih.gov/Blast.cgi). Multiple sequence alignments of protein were done by T-Coffee with default setting. After manual correction for poorly aligned region, the alignments format was changed to nexus. A phylogenetic tree of the sequenced lines was reconstructed by the neighbor-joining (NJ) method by MEGA3 [37]. Bootstrap values were estimated (with 1000 replicates) to assess the relative support for each branch. All positions containing alignment gaps were eliminated in pairwise 
sequence comparisons in $\mathrm{NJ}$ analyses.

\section{Subcellular localization of DEP2}

To determine localization of DEP2 protein in plant cells, fulllength $D E P 2$ coding sequence was amplified by PCR with primers 5'-AGA TCT GAT GGA GCC CGA CGC CCC G-3' and 5'CCT AGG CCT GAG CCT TGC ATC ACC-3', and inserted into the $B g l \mathrm{II} / \mathrm{SpeI}$ site of pCAMBIA1302 vector. The fusion construct and control were transformed into onion epidermis cells by particle bombardment using PDS-1000/He (BIO-RAD) [38]. This construct was also introduced into Agrobacterium tumefaciens GV3101 and then infiltrated into the leaves of 3-week-old Nicotiana benthamiana plants [39, 40]. GFP was detected by a confocal laser scanning microscopy (Leica TCS SP5) $24 \mathrm{~h}$ after transformation of onion epidermis cells and $48 \mathrm{~h}$ after infiltration of $N$. benthamiana leaves.

\section{Acknowledgments}

We thank Professor Gary Loake (University of Edinburg, UK) for critical reading of this manuscript. This work was supported by grants from Ministry of Agriculture of China (2008ZX08001), Ministry of Science and Technology of China (2009CB118506, 2006AA10A101), and National Natural Science Foundation of China (30671128, 30621001).

\section{References}

1 Wang Y, Li J. Molecular basis of plant architecture. Annu Rev Plant Biol 2008; 59:253-279.

2 Jin J, Huang W, Gao JP, et al. Genetic control of rice plant architecture under domestication. Nat Genet 2008; 40:13651369.

3 Li P, Wang Y, Qian Q, et al. LAZY1 controls rice shoot gravitropism through regulating polar auxin transport. Cell Res 2007; 17:402-410.

4 Tan L, Li X, Liu F, et al. Control of a key transition from prostrate to erect growth in rice domestication. Nat Genet 2008; 40:1360-1364.

$5 \mathrm{Yu} \mathrm{B}$, Lin Z, Li H, et al. TAC1, a major quantitative trait locus controlling tiller angle in rice. Plant J 2007; 52:891-898.

6 Sasaki A, Ashikari M, Ueguchi-Tanaka M, et al. Green revolution: a mutant gibberellin-synthesis gene in rice. Nature 2002; 416:701-702.

7 Spielmeyer W, Ellis MH, Chandler PM. Semidwarf (sd1), "green revolution" rice, contains a defective gibberellin 20-oxidase gene. Proc Natl Acad Sci USA 2002; 99:90439048.

8 Gao SJ, Chen WF, Zhang BL. Studies of erect panicle in rice. J Jilin Agric Sci 1999; 24:12-15.

9 Xu ZJ, Chen WF, Zhang LB, Yang SR. Design principles and parameters of rice ideal panicle type. Chin Sci Bull 2005; 50:2253-2256.

10 Huang X, Qian Q, Liu Z, et al. Natural variation at the DEP1 locus enhances grain yield in rice. Nat Genet 2009; 41:494497.

11 Kong FN, Wang JY, Zou JC, et al. Molecular tagging and mapping of the erect panicle gene in rice. Mol Breed 2007; 19:297-304.
12 Wang J, Nakazaki T, Chen S, et al. Identification and characterization of the erect-pose panicle gene EP conferring high grain yield in rice (Oryza sativa L.). Theor Appl Genet 2009; 119:85-91.

13 Yan CJ, Zhou JH, Yan S, et al. Identification and characterization of a major QTL responsible for erect panicle trait in japonica rice (Oryza sativa L.). Theor Appl Genet 2007; 115:1093-1100.

14 Zhou Y, Zhu J, Li Z, et al. Deletion in a quantitative trait gene qPE9-1 associated with panicle erectness improves plant architecture during rice domestication. Genetics 2009; 183:315324.

15 Piao R, Jiang W, Ham TH, et al. Map-based cloning of the ERECT PANICLE 3 gene in rice. Theor Appl Genet 2009; 119:1497-1506.

16 Zhou J, Xu Y, Xu P, et al. Introgression and mapping of erect panicle gene from Oryza glaberrima in to Oryza Sativa. Rice Genet Newsl 2008; 24:18-21.

17 Ikeda K, Sunohara H, Nagato Y. Developmental course of inflorescence and spikelet in rice. Breed Sci 2004; 54:147-156.

18 Ashikari M, Sakakibara H, Lin S, et al. Cytokinin oxidase regulates rice grain production. Science 2005; 309:741-745.

19 Ikeda K, Ito M, Nagasawa N, Kyozuka J, Nagato Y. Rice $A B$ ERRANT PANICLE ORGANIZATION 1, encoding an F-box protein, regulates meristem fate. Plant J 2007; 51:1030-1040.

20 Ikeda K, Nagasawa N, Itoh M, Kyozuka J, Nagato Y. Analyses of ABERRANT PANICLE ORGANIZATION 1 (APO1) gene regulating the spikelet number in rice. Plant Cell Physiol 2007; 48:S52-S52.

21 Komatsu K, Maekawa M, Ujiie S, et al. LAX and SPA: major regulators of shoot branching in rice. Proc Natl Acad Sci USA 2003; 100:11765-11770.

22 Komatsu M, Chujo A, Nagato Y, Shimamoto K, Kyozuka J. FRIZZY PANICLE is required to prevent the formation of axillary meristems and to establish floral meristem identity in rice spikelets. Development 2003; 130:3841-3850.

23 Li X, Qian Q, Fu Z, et al. Control of tillering in rice. Nature 2003; 422:618-621.

24 Suzaki T, Sato M, Ashikari M, Miyoshi M, Nagato Y, Hirano HY. The gene FLORAL ORGAN NUMBER1 regulates floral meristem size in rice and encodes a leucine-rich repeat receptor kinase orthologous to Arabidopsis CLAVATA1. Development 2004; 131:5649-5657.

25 Li S, Qian Q, Fu Z, et al. Short paniclel encodes a putative PTR family transporter and determines rice panicle size. Plant $J$ 2009; 58:592-605.

26 Ma Y, Liu L, Zhu C, et al. Molecular analysis of rice plants harboring a multi-functional T-DNA tagging system. J Genet Genomics 2009; 36:267-276.

27 Finn RD, Tate J, Mistry J, et al. The Pfam protein families database. Nucleic Acids Res 2008; 36:D281-288.

28 Yamamoto YY, Matsui M, Ang LH, Deng XW. Role of a COP1 interactive protein in mediating light-regulated gene expression in Arabidopsis. Plant Cell 1998; 10:1083-1094.

29 Dingkuhn M, Schnier HF, Dedatta SK, Dorffling K, Javellana C. Relationships between ripening-phase productivity and crop duration, canopy photosynthesis and senescence in transplanted and direct-seeded lowland Rice. Field Crops Res 1991; 26:327-345. 
30 Zhang WZ, Xu ZJ, Zhang LB, et al. Analysis on evolution for the erect panicle type varieties of rice. J Shenyang Agric Univ 2002; 33:161-166.

31 McCouch SR, Teytelman L, Xu Y, et al. Development and mapping of 2240 new SSR markers for rice (Oryza sativa L.) (supplement). DNA Res 2002; 9:257-279.

32 McCouch SR, Teytelman L, Xu Y, et al. Development and mapping of 2240 new SSR markers for rice (Oryza sativa L.). DNA Res 2002; 9:199-207.

33 Liu X, Bai X, Wang X, Chu C. OsWRKY71, a rice transcription factor, is involved in rice defense response. J Plant Physiol 2007; 164:969-979.

34 Luo A, Qian Q, Yin H, et al. EUI1, encoding a putative cytochrome P450 monooxygenase, regulates internode elongation by modulating gibberellin responses in rice. Plant Cell Physiol 2006; 47:181-191.

35 Chomczynski P, Sacchi N. Single-step method of RNA isolation by acid guanidinium thiocyanate-phenol-chloroform ex- traction. Anal Biochem 1987; 162:156-159.

36 Jefferson RA. The GUS reporter gene system. Nature 1989; 342:837-838.

37 Kumar S, Tamura K, Nei M. MEGA3: integrated software for molecular evolutionary genetics analysis and sequence alignment. Brief Bioinform 2004; 5:150-163.

38 Liu XQ, Bai XQ, Qian Q, Wang XJ, Chen MS, Chu CC. Os$W R K Y 03$, a rice transcriptional activator that functions in defense signaling pathway upstream of OsNPR1. Cell Res 2005; 15:593-603.

39 Sparkes IA, Runions J, Kearns A, Hawes C. Rapid, transient expression of fluorescent fusion proteins in tobacco plants and generation of stably transformed plants. Nat Protoc 2006; 1:2019-2025.

40 Voinnet O, Rivas S, Mestre P, Baulcombe D. An enhanced transient expression system in plants based on suppression of gene silencing by the $\mathrm{p} 19$ protein of tomato bushy stunt virus. Plant J 2003; 33:949-956.

(Supplementary information is linked to the online version of the paper on the Cell Research website.) 